\title{
Cambios en el modelo de enseñanza del metabolismo \\ a través de ciclos de mejora en el aula
}

\section{Changes in the teaching approach of metabolism through improvement cycles in classroom}

Sergio MontSerRat de LA PAZ

https://orcid.org/0000-0001-5400-3192

Departamento de Bioquímica Médica y

Biología Molecular e Inmunología.

Facultad de Medicina. Universidad de Sevilla

delapaz@us.es

DOI: http://dx.doi.org/10.12795/9788447231003.049

Pp.: 1026-1043 


\section{Contexto}

El Ciclo de Mejora en el Aula (CIMA) se desarrolló en la asignatura "Bioquímica del Metabolismo". Esta asignatura de formación básica (obligatoria) tiene 6 ECTS, de los cuales 4,5 ECTS son de teoría y los restantes 1,5 son prácticas de laboratorio. Se imparte en grupo único en el primer cuatrimestre del segundo curso del Grado en Biomedicina Básica y Experimental y tiene como objetivo general que los alumnos adquieran una visión global del metabolismo humano, sus principales rutas metabólicas y la regulación de estas. En el curso 2020/2021 se encontraban matriculados 44 alumnos (3 repetidores) y en este año, las clases teóricas se han impartido en sesiones de una hora de forma telemática síncrona a través de la plataforma de enseñanza virtual de la Universidad de Sevilla.

Es una asignatura muy conceptual y tradicionalmente este tipo de asignaturas se ha impartido a través de lecciones magistrales con muy escasa o nula participación del alumnado. Los alumnos suelen encontrar dificultades para estudiar las rutas bioquímicas del metabolismo, en concreto para comprender las relaciones entre las vías metabólicas y su integración porque estas vías siempre se enseñan individualmente en el aula.

El contenido de la asignatura se divide en 6 bloques temáticos y un total de 25 temas. Para la aplicación de este Ciclo de Mejora se eligió el bloque temático "Metabolismo de Lípidos". El objetivo de este bloque es que el alumno conozca y comprenda las principales rutas del metabolismo lipídico, su función en el organismo y su relación con distintas patologías.

Ciclos de Mejora en el Aula (2020). Experiencias de Innovación Docente de la US Esta obra se distribuye con la licencia Creative Commons 


\section{Diseño Previo del CIMA}

En esta sección se describe el diseño previo del CIMA, realizado antes de empezar su aplicación. Se incluye el mapa de contenido, el modelo metodológico posible, la secuencia de actividades programadas y el cuestionario que realizaron los alumnos antes y después del CIMA.

\section{Mapa de Contenidos}

El mapa de contenidos se presenta en la Figura 1. El problema en torno al cual se construye es la función del metabolismo lipídico. Por ello empezamos preguntándonos qué hacen las grasas en nuestro cuerpo (recuadro azul oscuro) y se identificaron las ideas principales (recuadros amarillos). El mapa de contenidos permitió generar un hilo conductor que explique la relación entre los diferentes conceptos y su implicación en la aparición de diversas patologías (recuadros rojos). Como se puede apreciar en el mapa, en este ciclo se incluyen contenidos conceptuales y procedimentales, datos y valores.

METABOLISMO LIPÍDICO

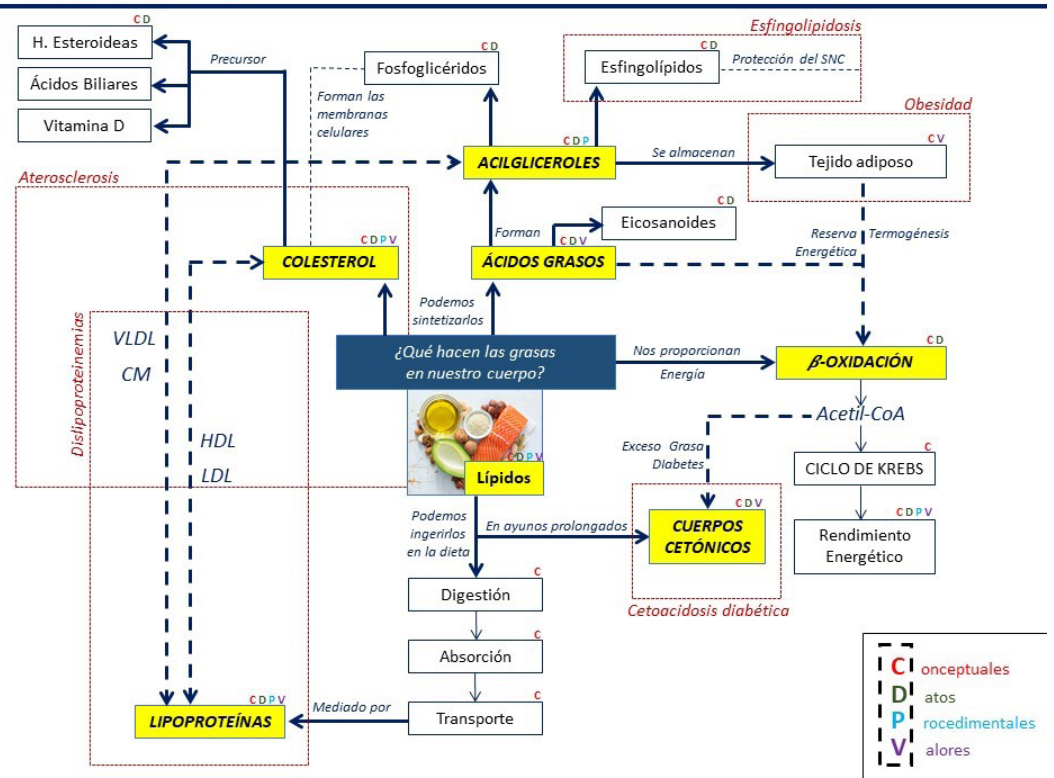

Figura 1. Mapa de contenido. Ciclos de Mejora en el Aula (2020). Experiencias de Innovación Docente de la US Reconocimiento-NoComercial-SinObraDerivada Internacional (CC BY-NC-ND 4.0.) 


\section{Modelo metodológico posible}

En la Figura 2 se presenta el modelo metodológico posible con el que se pretende desarrollar el CIMA. Como se muestra en la figura, habrá una primera sesión (S1) que constará de 2 actividades, una primera de Introducción al CIMA y al cuestionario (I) y una segunda en la que los alumnos responderán el cuestionario inicial (Qi). Las siguientes sesiones (S2-S7) se iniciarán con un repaso (R) de lo visto en la sesión anterior y plantearemos una serie de Preguntas (P). Los alumnos tendrán un tiempo para la reflexión y el trabajo en grupo (IA) y posteriormente se abrirá un tiempo de debate/discusión (D) a partir de cual iremos construyendo los contenidos mediante actividades de contraste (AC) previstos para la sesión (de Alba y Porlan, 2017). Tras finalizar dicha fase realizaremos el repaso de la sesión construyendo un mapa de contenido (MC). La última sesión (S8) constará de 2 actividades, la primera será la construcción de un mapa de contenido del bloque temático y una segunda actividad que consistirá en responder al cuestionario final (Qf).

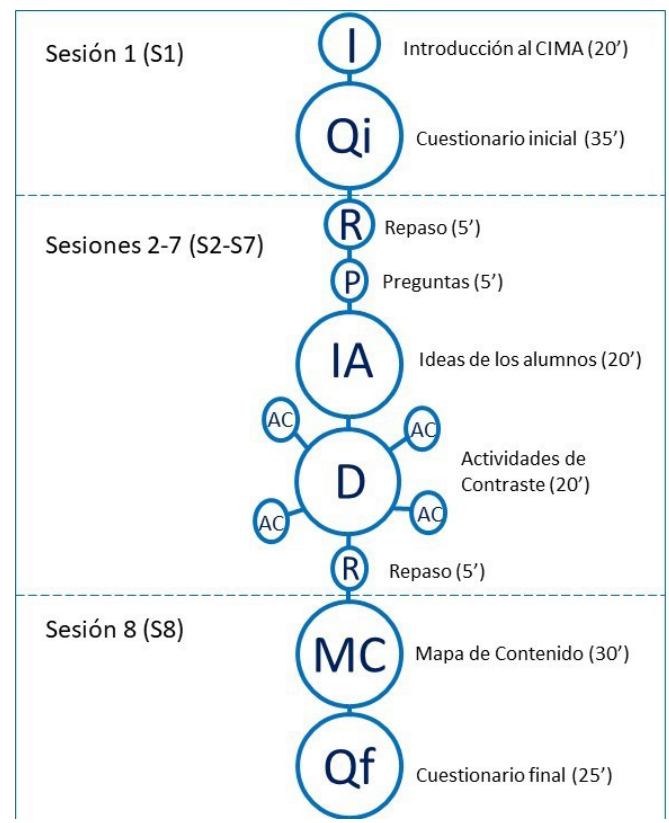

Figura 2. Modelo metodológico posible.

Ciclos de Mejora en el Aula (2020). Experiencias de Innovación Docente de la US Esta obra se distribuye con la licencia Creative Commons 


\section{Secuencia de Actividades}

Las actividades diseñadas para este ciclo de mejora han seguido el patrón básico de los "talleres conceptuales" propuestos por Finkel (2008), en los que se les proporcionan a los estudiantes los cuatro elementos fundamentales para acompañarles en el proceso de aprendizaje: 1) otra persona con la que compartir el proceso mediante la realización de trabajo en grupo; 2) una secuencia de preguntas bien diseñada que le fuerza a "investigar" y generar hipótesis en torno a un mismo hilo conductor; 3 ) un profesor al que puede acudir para solventar pequeñas dudas que le obstaculicen el proceso; d) un desenlace intelectual que le permita evaluar los resultados de su trabajo compartiéndolo con sus compañeros y compañeras. A continuación, en la Tabla 1 se muestra de forma resumida la secuencia de actividades diseñada para el CIMA.

Tabla 1. Secuencia de actividades.

\section{Sesión 1 (55 minutos)}

\begin{tabular}{|c|c|l|c|c|}
\hline $\begin{array}{c}\text { No } \\
\text { Actividad }\end{array}$ & Nombre & \multicolumn{1}{|c|}{ Descripción } & $\begin{array}{l}\text { Tiempo } \\
\text { Previsto }\end{array}$ & $\begin{array}{c}\text { Contenidos } \\
\text { abordados }\end{array}$ \\
\hline $\mathbf{1}$ & $\begin{array}{l}\text { Introducción } \\
\text { al CIMA }\end{array}$ & $\begin{array}{l}\text { Se informará a los } \\
\text { alumnos sobre el CIMA y la } \\
\text { metodología que se utilizará, } \\
\text { así como la dinámica de las } \\
\text { sesiones. }\end{array}$ & 20 min & - \\
\hline $\mathbf{2}$ & $\begin{array}{l}\text { Cuestionario } \\
\text { Inicial }\end{array}$ & $\begin{array}{l}\text { Se habilitará en la plataforma } \\
\text { virtual el cuestionario con } \\
\text { 5 preguntas abiertas que } \\
\text { responderán de manera } \\
\text { individual }\end{array}$ & 35 min & - \\
\hline
\end{tabular}

Ciclos de Mejora en el Aula (2020). Experiencias de Innovación Docente de la US Esta obra se distribuye con la licencia Creative Commons 
Sesión 2 (55 minutos)

\begin{tabular}{|c|c|c|c|c|}
\hline $\begin{array}{c}\text { No } \\
\text { Actividad }\end{array}$ & Nombre & Descripción & $\begin{array}{l}\text { Tiempo } \\
\text { Previsto }\end{array}$ & Contenidos abordados \\
\hline 1 & Repaso & $\begin{array}{l}\text { Se realizará un repaso } \\
\text { de la dinámica de la } \\
\text { sesión y de contenidos } \\
\text { de Bioquímica } \\
\text { Estructural (Asignatura } \\
\text { de primer curso del } \\
\text { grado) }\end{array}$ & $5 \mathrm{~min}$ & $\begin{array}{l}\text { Bioquímica Estructural } \\
\text { (Estructura de Lípidos) }\end{array}$ \\
\hline 2 & Preguntas & $\begin{array}{l}\text { Se compartirá un } \\
\text { archivo con } 3 \text { preguntas } \\
\text { sobre los contenidos } \\
\text { estructurantes previstos } \\
\text { para la sesión. }\end{array}$ & $5 \mathrm{~min}$ & \multirow{4}{*}{$\begin{array}{l}\text { Ingesta y transporte de } \\
\text { lípidos en sangre. } \\
\text { Lipoproteínas } \\
\text { plasmáticas. } \\
\text { Clasificación y aspectos } \\
\text { estructurales. } \\
\text { Metabolismo de las } \\
\text { lipoproteínas. } \\
\text { Dislipoproteinemias. }\end{array}$} \\
\hline 3 & $\begin{array}{l}\text { Ideas de los } \\
\text { estudiantes }\end{array}$ & $\begin{array}{l}\text { Los alumnos deber } \\
\text { reflexionar sobre las } \\
\text { preguntas planteadas, } \\
\text { puede utilizar internet } \\
\text { para abordarlas. Los } \\
\text { grupos se formarán } \\
\text { en cada sesión por el } \\
\text { profesor y cada alumno } \\
\text { entrará en la sesión de } \\
\text { blackboard específica } \\
\text { de su grupo }\end{array}$ & $20 \mathrm{~min}$ & \\
\hline 4 & $\begin{array}{l}\text { Debate/ } \\
\text { Actividades } \\
\text { de } \\
\text { contraste }\end{array}$ & $\begin{array}{l}\text { Una vez concluido el } \\
\text { tiempo de reflexión, los } \\
\text { alumnos volverán a la } \\
\text { sesión de blackboard } \\
\text { general del curso. } \\
\text { Se abre un debate } \\
\text { para entre todos ir } \\
\text { construyendo los } \\
\text { contenidos previstos } \\
\text { para la sesión }\end{array}$ & $20 \mathrm{~min}$ & \\
\hline 5 & Repaso & $\begin{array}{l}\text { Se construye entre } \\
\text { todos en la pizarra un } \\
\text { resumen a modo de } \\
\text { mapa de contenidos }\end{array}$ & $5 \mathrm{~min}$ & \\
\hline
\end{tabular}

Ciclos de Mejora en el Aula (2020). Experiencias de Innovación Docente de la US Esta obra se distribuye con la licencia Creative Commons 
Sesión 3 (55 minutos)

\begin{tabular}{|c|c|c|c|c|}
\hline $\begin{array}{c}\text { No } \\
\text { Actividad }\end{array}$ & Nombre & Descripción & $\begin{array}{l}\text { Tiempo } \\
\text { Previsto }\end{array}$ & Contenidos abordados \\
\hline 1 & Repaso & $\begin{array}{l}\text { Se realizará un repaso } \\
\text { de la sesión anterior }\end{array}$ & $5 \mathrm{~min}$ & $\begin{array}{l}\text { Contenidos vistos } \\
\text { en la sesión anterior } \\
\text { correspondiente } \\
\text { al bloque temático } \\
\text { "Metabolismo de } \\
\text { Lípidos" }\end{array}$ \\
\hline 2 & Preguntas & $\begin{array}{l}\text { Se compartirá } \\
\text { un archivo con } 3 \\
\text { preguntas sobre } \\
\text { los contenidos } \\
\text { estructurantes } \\
\text { previstos para la } \\
\text { sesión. }\end{array}$ & $5 \mathrm{~min}$ & \multirow{4}{*}{$\begin{array}{l}\text { Metabolismo de los } \\
\text { triglicéridos. } \\
\text { Lipolisis: Oxidación } \\
\text { de los ácidos grasos: } \\
\text { activación, entrada } \\
\text { en la mitocondria y } \\
\beta \text {-oxidación. } \\
\text { Regulación metabólica } \\
\text { y hormonal. } \\
\text { Oxidación de ácidos } \\
\text { grasos insaturados y } \\
\text { de número impar de } \\
\text { átomos de carbono. }\end{array}$} \\
\hline 3 & $\begin{array}{l}\text { Ideas de los } \\
\text { estudiantes }\end{array}$ & $\begin{array}{l}\text { Los alumnos deber } \\
\text { reflexionar sobre las } \\
\text { preguntas planteadas, } \\
\text { puede utilizar internet } \\
\text { para abordarlas. Los } \\
\text { grupos se formarán } \\
\text { en cada sesión por } \\
\text { el profesor y cada } \\
\text { alumno entrará en la } \\
\text { sesión de blackboard } \\
\text { específica de su grupo }\end{array}$ & $20 \mathrm{~min}$ & \\
\hline 4 & $\begin{array}{c}\text { Debate/ } \\
\text { Actividades } \\
\text { de contraste }\end{array}$ & $\begin{array}{l}\text { Una vez concluido el } \\
\text { tiempo de reflexión, } \\
\text { los alumnos volverán } \\
\text { a la sesión de } \\
\text { blackboard general } \\
\text { del curso. Se abre un } \\
\text { debate para entre } \\
\text { todos ir construyendo } \\
\text { los contenidos } \\
\text { previstos para la } \\
\text { sesión }\end{array}$ & $20 \min$ & \\
\hline 5 & Repaso & $\begin{array}{l}\text { Se construye entre } \\
\text { todos en la pizarra un } \\
\text { resumen a modo de } \\
\text { mapa de contenidos }\end{array}$ & $5 \mathrm{~min}$ & \\
\hline
\end{tabular}

Ciclos de Mejora en el Aula (2020). Experiencias de Innovación Docente de la US Esta obra se distribuye con la licencia Creative Commons Reconocimiento-NoComercial-SinObraDerivada Internacional (CC BY-NC-ND 4.0.) 
Sesión 4 (55 minutos)

\begin{tabular}{|c|c|c|c|c|}
\hline $\begin{array}{c}\text { No } \\
\text { Actividad }\end{array}$ & Nombre & Descripción & $\begin{array}{l}\text { Tiempo } \\
\text { Previsto }\end{array}$ & $\begin{array}{l}\text { Contenidos } \\
\text { abordados }\end{array}$ \\
\hline 1 & Repaso & $\begin{array}{l}\text { Se realizará un repaso de } \\
\text { la sesión anterior }\end{array}$ & $5 \mathrm{~min}$ & $\begin{array}{l}\text { Contenidos } \\
\text { vistos en la } \\
\text { sesión anterior } \\
\text { correspondiente } \\
\text { al bloque temático } \\
\text { "Metabolismo de } \\
\text { Lípidos" }\end{array}$ \\
\hline 2 & Preguntas & $\begin{array}{l}\text { Se compartirá un } \\
\text { archivo con } 3 \text { preguntas } \\
\text { sobre los contenidos } \\
\text { estructurantes previstos } \\
\text { para la sesión. }\end{array}$ & $5 \mathrm{~min}$ & \multirow{4}{*}{$\begin{array}{l}\text { Cuerpos cetónicos. } \\
\text { Etapas en } \\
\text { la síntesis y } \\
\text { utilización de } \\
\text { cuerpos cetónicos. } \\
\text { Relación } \\
\text { metabólica entre } \\
\text { glucosa, ácidos } \\
\text { grasos y cuerpos } \\
\text { cetónicos. }\end{array}$} \\
\hline 3 & $\begin{array}{l}\text { Ideas de los } \\
\text { estudiantes }\end{array}$ & $\begin{array}{l}\text { Los alumnos deber } \\
\text { reflexionar sobre las } \\
\text { preguntas planteadas, } \\
\text { puede utilizar internet } \\
\text { para abordarlas. Los } \\
\text { grupos se formarán } \\
\text { en cada sesión por el } \\
\text { profesor y cada alumno } \\
\text { entrará en la sesión de } \\
\text { blackboard específica de } \\
\text { su grupo }\end{array}$ & $20 \min$ & \\
\hline 4 & $\begin{array}{c}\text { Debate/ } \\
\text { Actividades } \\
\text { de contraste }\end{array}$ & $\begin{array}{l}\text { Una vez concluido el } \\
\text { tiempo de reflexión, los } \\
\text { alumnos volverán a la } \\
\text { sesión de blackboard } \\
\text { general del curso. } \\
\text { Se abre un debate } \\
\text { para entre todos ir } \\
\text { construyendo los } \\
\text { contenidos previstos } \\
\text { para la sesión }\end{array}$ & $20 \min$ & \\
\hline 5 & Repaso & $\begin{array}{l}\text { Se construye entre todos } \\
\text { en la pizarra un resumen } \\
\text { a modo de mapa de } \\
\text { contenidos }\end{array}$ & $5 \mathrm{~min}$ & \\
\hline
\end{tabular}

Ciclos de Mejora en el Aula (2020). Experiencias de Innovación Docente de la US Esta obra se distribuye con la licencia Creative Commons Reconocimiento-NoComercial-SinObraDerivada Internacional (CC BY-NC-ND 4.0.) 
Sesión 5 (55 minutos)

\begin{tabular}{|c|c|c|c|c|}
\hline $\begin{array}{c}\text { № } \\
\text { Actividad }\end{array}$ & Nombre & Descripción & $\begin{array}{l}\text { Tiempo } \\
\text { Previsto }\end{array}$ & $\begin{array}{l}\text { Contenidos } \\
\text { abordados }\end{array}$ \\
\hline 1 & Repaso & $\begin{array}{l}\text { Se realizará un repaso } \\
\text { de la sesión anterior }\end{array}$ & $5 \mathrm{~min}$ & $\begin{array}{l}\text { Contenidos vistos } \\
\text { en la sesión anterior } \\
\text { correspondiente } \\
\text { al bloque temático } \\
\text { "Metabolismo de } \\
\text { Lípidos" }\end{array}$ \\
\hline 2 & Preguntas & $\begin{array}{l}\text { Se compartirá un } \\
\text { archivo con } 3 \text { preguntas } \\
\text { sobre los contenidos } \\
\text { estructurantes } \\
\text { previstos para la } \\
\text { sesión. }\end{array}$ & $5 \mathrm{~min}$ & \multirow{4}{*}{$\begin{array}{l}\text { Biosíntesis de ácidos } \\
\text { grasos. } \\
\text { Formación de } \\
\text { malonil-CoA. } \\
\text { Complejo enzimático } \\
\text { de la sintetasa de } \\
\text { ácido graso. } \\
\text { Elongación de ácidos } \\
\text { grasos. } \\
\text { Formación de ácidos } \\
\text { grasos insaturados. }\end{array}$} \\
\hline 3 & $\begin{array}{l}\text { Ideas de los } \\
\text { estudiantes }\end{array}$ & $\begin{array}{l}\text { Los alumnos deber } \\
\text { reflexionar sobre las } \\
\text { preguntas planteadas, } \\
\text { puede utilizar internet } \\
\text { para abordarlas. Los } \\
\text { grupos se formarán } \\
\text { en cada sesión por el } \\
\text { profesor y cada alumno } \\
\text { entrará en la sesión de } \\
\text { blackboard específica } \\
\text { de su grupo }\end{array}$ & $20 \mathrm{~min}$ & \\
\hline 4 & $\begin{array}{c}\text { Debate/ } \\
\text { Actividades } \\
\text { de } \\
\text { contraste }\end{array}$ & $\begin{array}{l}\text { Una vez concluido el } \\
\text { tiempo de reflexión, los } \\
\text { alumnos volverán a la } \\
\text { sesión de blackboard } \\
\text { general del curso. } \\
\text { Se abre un debate } \\
\text { para entre todos ir } \\
\text { construyendo los } \\
\text { contenidos previstos } \\
\text { para la sesión } \\
\end{array}$ & $20 \mathrm{~min}$ & \\
\hline 5 & Repaso & $\begin{array}{l}\text { Se construye entre } \\
\text { todos en la pizarra un } \\
\text { resumen a modo de } \\
\text { mapa de contenidos }\end{array}$ & $5 \mathrm{~min}$ & \\
\hline
\end{tabular}

Ciclos de Mejora en el Aula (2020). Experiencias de Innovación Docente de la US Esta obra se distribuye con la licencia Creative Commons Reconocimiento-NoComercial-SinObraDerivada Internacional (CC BY-NC-ND 4.0.) 
Sesión 6 (55 minutos)

\begin{tabular}{|c|c|c|c|c|}
\hline $\begin{array}{c}\text { № } \\
\text { Actividad }\end{array}$ & Nombre & Descripción & $\begin{array}{l}\text { Tiempo } \\
\text { Previsto }\end{array}$ & $\begin{array}{l}\text { Contenidos } \\
\text { abordados }\end{array}$ \\
\hline 1 & Repaso & $\begin{array}{l}\text { Se realizará un repaso } \\
\text { de la sesión anterior }\end{array}$ & $5 \mathrm{~min}$ & $\begin{array}{l}\text { Contenidos vistos } \\
\text { en la sesión anterior } \\
\text { correspondiente } \\
\text { al bloque temático } \\
\text { "Metabolismo de } \\
\text { Lípidos" }\end{array}$ \\
\hline 2 & Preguntas & $\begin{array}{l}\text { Se compartirá } \\
\text { un archivo con } 3 \\
\text { preguntas sobre } \\
\text { los contenidos } \\
\text { estructurantes } \\
\text { previstos para la } \\
\text { sesión. }\end{array}$ & $5 \mathrm{~min}$ & \multirow{4}{*}{$\begin{array}{l}\text { Lípidos complejos: } \\
\text { fosfoglicéridos y } \\
\text { esfingolípidos. } \\
\text { Lípidos simples: } \\
\text { terpenos, esteroides, } \\
\text { prostaglandinas y } \\
\text { carotinoides. } \\
\text { Sintesis y } \\
\text { transformaciones de } \\
\text { los eicosanoides. } \\
\text { Actividad } \\
\text { prostaglandina } \\
\text { sintetasa. }\end{array}$} \\
\hline 3 & $\begin{array}{l}\text { Ideas de los } \\
\text { alumnos }\end{array}$ & $\begin{array}{l}\text { Los alumnos deber } \\
\text { reflexionar sobre las } \\
\text { preguntas planteadas, } \\
\text { puede utilizar internet } \\
\text { para abordarlas. Los } \\
\text { grupos se formarán } \\
\text { en cada sesión por } \\
\text { el profesor y cada } \\
\text { alumno entrará en la } \\
\text { sesión de blackboard } \\
\text { específica de su grupo }\end{array}$ & $20 \mathrm{~min}$ & \\
\hline 4 & $\begin{array}{c}\text { Debate/ } \\
\text { Actividades } \\
\text { de contraste }\end{array}$ & $\begin{array}{l}\text { Una vez concluido el } \\
\text { tiempo de reflexión, los } \\
\text { alumnos volverán a la } \\
\text { sesión de blackboard } \\
\text { general del curso. } \\
\text { Se abre un debate } \\
\text { para entre todos ir } \\
\text { construyendo los } \\
\text { contenidos previstos } \\
\text { para la sesión }\end{array}$ & $20 \mathrm{~min}$ & \\
\hline 5 & Repaso & $\begin{array}{l}\text { Se construye entre } \\
\text { todos en la pizarra un } \\
\text { resumen a modo de } \\
\text { mapa de contenidos }\end{array}$ & $5 \mathrm{~min}$ & \\
\hline
\end{tabular}

Ciclos de Mejora en el Aula (2020). Experiencias de Innovación Docente de la US Esta obra se distribuye con la licencia Creative Commons 
Sesión 7 (55 minutos)

\begin{tabular}{|c|c|c|c|c|}
\hline $\begin{array}{c}\text { № } \\
\text { Actividad }\end{array}$ & Nombre & Descripción & $\begin{array}{l}\text { Tiempo } \\
\text { Previsto }\end{array}$ & Contenidos abordados \\
\hline 1 & Repaso & $\begin{array}{l}\text { Se realizará un repaso } \\
\text { de la sesión anterior }\end{array}$ & $5 \mathrm{~min}$ & $\begin{array}{l}\text { Contenidos vistos } \\
\text { en la sesión anterior } \\
\text { correspondiente } \\
\text { al bloque temático } \\
\text { "Metabolismo de } \\
\text { Lípidos" }\end{array}$ \\
\hline 2 & Preguntas & $\begin{array}{l}\text { Se compartirá un } \\
\text { archivo con } 3 \text { preguntas } \\
\text { sobre los contenidos } \\
\text { estructurantes previstos } \\
\text { para la sesión. }\end{array}$ & $5 \mathrm{~min}$ & \multirow{4}{*}{$\begin{array}{l}\text { Metabolismo del } \\
\text { colesterol. } \\
\text { Biosíntesis y } \\
\text { degradación del } \\
\text { colesterol. } \\
\text { Regulación de la } \\
\text { actividad de la HMG- } \\
\text { CoA reductasa. } \\
\text { Ácidos y sales biliares } \\
\text { biliares. } \\
\text { Aterogénesis. Origen y } \\
\text { desarrollo del proceso } \\
\text { ateroesclerótico. }\end{array}$} \\
\hline 3 & $\begin{array}{l}\text { Ideas de los } \\
\text { alumnos }\end{array}$ & $\begin{array}{l}\text { Los alumnos deber } \\
\text { reflexionar sobre las } \\
\text { preguntas planteadas, } \\
\text { puede utilizar internet } \\
\text { para abordarlas. Los } \\
\text { grupos se formarán } \\
\text { en cada sesión por el } \\
\text { profesor y cada alumno } \\
\text { entrará en la sesión de } \\
\text { blackboard específica } \\
\text { de su grupo }\end{array}$ & $20 \mathrm{~min}$ & \\
\hline 4 & $\begin{array}{c}\text { Debate/ } \\
\text { Actividades } \\
\text { de contraste }\end{array}$ & $\begin{array}{l}\text { Una vez concluido el } \\
\text { tiempo de reflexión, los } \\
\text { alumnos volverán a la } \\
\text { sesión de blackboard } \\
\text { general del curso. } \\
\text { Se abre un debate } \\
\text { para entre todos ir } \\
\text { construyendo los } \\
\text { contenidos previstos } \\
\text { para la sesión }\end{array}$ & $20 \mathrm{~min}$ & \\
\hline 5 & Repaso & $\begin{array}{l}\text { Se construye entre } \\
\text { todos en la pizarra un } \\
\text { resumen a modo de } \\
\text { mapa de contenidos }\end{array}$ & $5 \mathrm{~min}$ & \\
\hline
\end{tabular}

Ciclos de Mejora en el Aula (2020). Experiencias de Innovación Docente de la US Esta obra se distribuye con la licencia Creative Commons Reconocimiento-NoComercial-SinObraDerivada Internacional (CC BY-NC-ND 4.0.) 
Sesión 8 (55 minutos)

\begin{tabular}{|c|l|l|l|l|}
\hline $\begin{array}{c}\text { No } \\
\text { Actividad }\end{array}$ & Nombre & Descripción & $\begin{array}{l}\text { Tiempo } \\
\text { Previsto }\end{array}$ & $\begin{array}{l}\text { Contenidos } \\
\text { abordados }\end{array}$ \\
\hline $\mathbf{1}$ & $\begin{array}{l}\text { Mapa de } \\
\text { Contenido }\end{array}$ & $\begin{array}{l}\text { Se elaborará entre todos } \\
\text { un mapa de contenido } \\
\text { global del bloque } \\
\text { temático a modo resumen } \\
\text { e interrelacionando todos } \\
\text { los contenidos abordados. }\end{array}$ & $30 \mathrm{~min}$ & $\begin{array}{l}\text { Metabolismo de } \\
\text { Lípidos }\end{array}$ \\
\hline $\mathbf{2}$ & $\begin{array}{l}\text { Cuestionario } \\
\text { Final }\end{array}$ & $\begin{array}{l}\text { Se habilitará en la } \\
\text { plataforma virtual el } \\
\text { mismo cuestionario que } \\
\text { se habilitó al principio } \\
\text { del CimA con 5 preguntas } \\
\text { abiertas que responderán } \\
\text { de manera individual }\end{array}$ & 25 min & - \\
\hline
\end{tabular}

\section{Cuestionario inicial-final}

Para evaluar los conocimientos previos de los alumnos sobre el tema, y como una herramienta de detección de posibles errores conceptuales, se pidió a los alumnos que respondieran a un breve cuestionario. Los resultados obtenidos se analizaron posteriormente mediante la creación de escaleras de aprendizaje. El cuestionario diseñado para los estudiantes contuvo cinco preguntas. Estas preguntas son cercanas, abiertas y estimulantes que dan información acerca de los modelos mentales del alumnado, ya que permiten responder con sus propios argumentos (Rivero y Porlán, 2017). Las preguntas fueron: 1) ¿Cómo viajan las grasas por la sangre? 2) ¿Qué engorda más $1 \mathrm{Kg}$ de grasa o $1 \mathrm{Kg}$ de azúcar? 3) ¿Cómo es posible que los osos hibernen? 4) ¿Qué son y para qué sirven las dietas cetogénicas? y 5) ¿Qué relación hay entre la testosterona y la vitamina D?

Ciclos de Mejora en el Aula (2020). Experiencias de Innovación Docente de la US Esta obra se distribuye con la licencia Creative Commons 


\section{Aplicación del CIMA}

\section{Descripción y desarrollo de las sesiones}

Se presenta una descripción resumida de las sesiones. Antes de iniciar el CIMA estaba nervioso por la aceptación de los alumnos y preocupado por si iba a saber gestionar los tiempos. En la Sesión 1 comencé con una breve introducción, donde les comenté que para este bloque temático tenía preparada una nueva forma de afrontar la asignatura que incluía una serie de actividades que esperaba les motivaran, y les pedí el compromiso de participación. Durante 30 minutos le comenté como sería la dinámica de las próximas 6 sesiones. Tras esta introducción al CIMA, les solicité que rellenaran un cuestionario que me permitiría evaluar la aplicación del CIMA. Este cuestionario constaba de las 5 preguntas indicadas anteriormente y estaba disponible en la carpeta de contenidos de la enseñanza virtual de la asignatura. Les pedí que una vez finalizado, tenían 30 minutos para completarlo, lo enviasen por correo a otra profesora, que se encargaría de codificar los nombres, para que la evaluación de las respuestas fuera a ciegas. Hice especial énfasis en que lo realizaran individualmente con el máximo de responsabilidad, reflexión y compromiso. Que no se apoyaran ni buscaran las respuestas en internet. Recibimos 44 cuestionarios. Al día siguiente estaba más nervioso aún, quería que todo saliese bien. En la Sesión 2, hago un breve resumen de la sesión anterior y recordé los puntos más destacables de la metodología del CIMA. A continuación, pongo en la pantalla compartida el enunciado de la primera pregunta con la que vamos a trabajar y posteriormente una serie 3 preguntas relacionadas con ella. Los alumnos pasan a unirse a los distintos grupos creados en la sesión. Aquí se crea un pequeño caos, pues al poder unirse libremente a cada grupo, había algunos alumnos que no sabían a cuál unirse, o no sabían como hacerlo. Se perdió mucho tiempo. Yo me fui pasando por los distintos grupos, mientras ellos estaban

Ciclos de Mejora en el Aula (2020). Experiencias de Innovación Docente de la US Esta obra se distribuye con la licencia Creative Commons 
discutiendo las distintas cuestiones. Esto, sin duda, fue lo más gratificante, verlos trabajar. Me llamó la atención que trabajaban de forma muy autónoma y con mucho interés. Estaban previstos 20 minutos, pero al volver a la sesión general todos los alumnos se quejaban de que faltaba más tiempo para profundizar en las cuestiones. Pasado este tiempo todos tenían que volver a la sesión general, aquí también se perdió algo de tiempo pues tuve que ir grupo a grupo recordándolo. Una vez todos en la sesión general, los alumnos empezaron a exponer sus ideas sobre las preguntas que les había lanzado al principio de la clase. Aunque faltaban algunos conceptos, en general estaban bastante bien fundamentadas las respuestas. Les comenté que una vez finalizada la clase le subiría un documento con todo el contenido de la sesión y acabamos los últimos 5 minutos proyectando una imagen del mapa de contenidos visto en la sesión. Al acabar la clase, sentí que había ido de forma muy acelerada, pensando que quizás el modelo diseñado no era el ideal para clases de 55 minutos. Durante la sesión 3 a la sesión 7 la dinámica fue muy similar a la sesión 2, comenzaba con un breve resumen de la clase anterior y compartía en la pantalla la pregunta clave y las otras 3 cuestiones que se derivan de ella. Cada vez la formación de los grupos se realizaba de forma más eficiente y la gestión del tiempo iba mejorando. A medida que avanzaron las sesiones me di cuenta que algunos grupos habían optado por dividirse las preguntas, y les recordé que no era el objetivo de la actividad. En general las sesiones transcurrieron sin incidentes y de manera satisfactoria para mi y para el alumnado como me reflejaron en alguna tutoría individual que tuve con algún alumno durante la realización del CIMA. La última sesión, la sesión 8, pedí un feedback rápido sobre que les había parecido el CIMA, y aunque en general les había gustado la dinámica, la mayoría preferían el modelo tradicional que había seguido en los bloques temáticos previos. La verdad que fue algo decepcionante después de tanto trabajo y estrés, pero me lo esperaba, mi modelo propuesto estaba demasiado ajustado en tiempo, con demasiados contenidos relevantes para tampoco tiempo de clase. A continuación, fui

Ciclos de Mejora en el Aula (2020). Experiencias de Innovación Docente de la US Esta obra se distribuye con la licencia Creative Commons 
proyectando en la pantalla un mapa de contenidos que se iba construyendo poco a poco y que servía a modo de resumen de todo el bloque temático. Los últimos 30 minutos los reservé para que volvieran a responder al mismo cuestionario inicial y lo enviasen por correo a mi compañera.

\section{Evaluación del aprendizaje de los estudiantes}

Tras la aplicación del CIMA se analizan los cuestionarios iniciales y finales para evaluar el aprendizaje de los alumnos. A modo de ejemplo, en la Figura $\mathbf{3}$ se presentan los resultados obtenidos en la pregunta 1 del cuestionario en forma de escalera. En el cuestionario inicial, el 28\% de los alumnos está en el escalón bajo, no han contestado a la pregunta o lo han hecho de forma errónea. En el escalón superior, un 63\% ha respondido de forma correcta pero incompleta y sin profundidad en los razonamientos. Solo un 9\% alcanza el escalón superior, contestado de forma razonada y precisa. Como se puede observar en la Figura 3, tras la aplicación del CIMA los alumnos han mejorado su capacidad para reflexionar sobre la pregunta dada, llegando a alcanzar el 56\% de ellos el escalón superior.

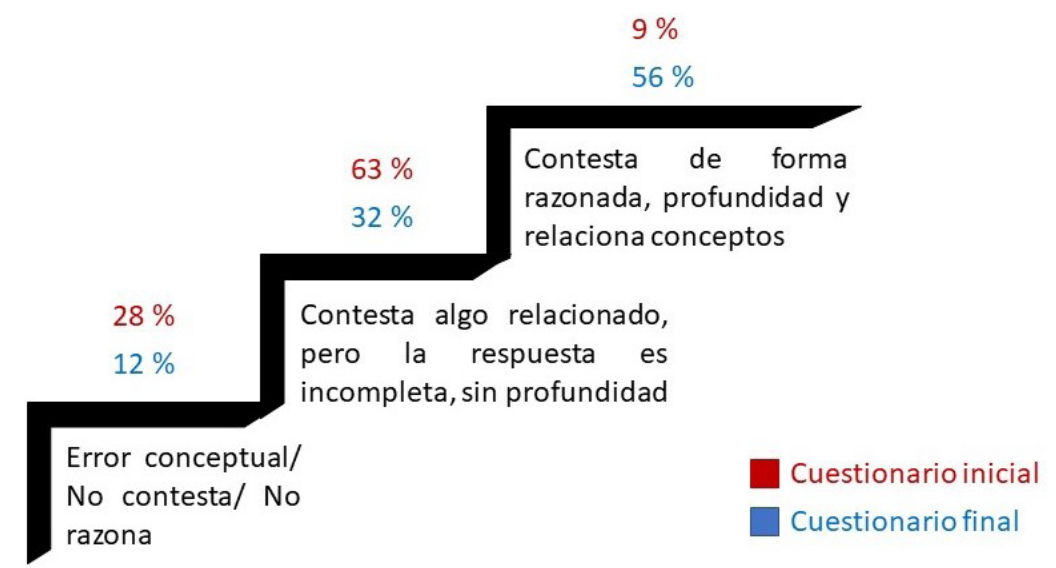

Figura 3. Escalera de aprendizaje a la pregunta 1 del cuestionario inicial y final “Cómo viajan las grasas por la sangre?".

Ciclos de Mejora en el Aula (2020). Experiencias de Innovación Docente de la US Esta obra se distribuye con la licencia Creative Commons 


\section{Evaluación del CIMA}

El CIMA me ha hecho afianzar algunas de las dinámicas que solía utilizar en mis clases, y mejorarlas. Por ejemplo, empezar cada tema con el planteamiento de una cuestión del día a día que pueda ser de interés para los alumnos y relacionado con los contenidos que vamos a tratar. Otro de los aspectos positivos de un modelo como el propuesto es que los alumnos van construyendo su propio aprendizaje a su ritmo no al que imponemos nosotros como profesor en las lecciones magistrales. También considero esencial los últimos 5-10 minutos donde nos paramos y hacemos un resumen de lo visto en la sesión, a través de un pequeño mapa de contenidos hace que alumno visualice de forma sencilla lo trabajado en el día. Por último, el compromiso (Bain, 2007), sin el es imposible llevar a cabo este tipo de docencia, compromiso del profesor por lograr que sus alumnos aprendan y compromiso de los alumnos a trabajar en el correcto desarrollo de las sesiones. En mi caso, mis alumnos han mostrado una actitud inmejorable. La principal dificultad detectada es la falta de tiempo para que el alumno profundice en las respuestas que tienen que resolver. Debido a ello asumimos que el material que con posterioridad le subimos a la enseñanza virtual con los contenidos de la sesión lo van a trabajar, y no a memorizar sin más. Como he comentado anteriormente, creo que ha fallado el modelo propuesto al tratarse de una asignatura en la que se imparten la teoría en sesiones de 1 hora y creo que sería óptimo sesiones de 2 horas. Además, es una asignatura muy conceptual con un gran volumen de contenido relevante y los alumnos consideran que tiene muchos conceptos que prepararse en casa de forma autónoma. Esto último no me preocupa, pues saben que cuentan conmigo para ir resolviendo las dudas que se le vayan planteando, pero si me inquieta haber ido tan acelerado durante las sesiones sin haberlas disfrutado al 100\% por la preocupación por ajustarme al tiempo previsto. Por

Ciclos de Mejora en el Aula (2020). Experiencias de Innovación Docente de la US Esta obra se distribuye con la licencia Creative Commons 
último, me gustaría indicar (para no olvidarme) que debemos hacer a los alumnos más independientes, más autónomos, que ellos sean capaces de generar sus contenidos, de crear su aprendizaje. Con todo ello, algunos de los modelos didácticos personales extraídos de la realización del CIMA son:

1. La mejor docencia crea una sensación de que todo el mundo está trabajando conjuntamente. El aprendizaje crítico natural compromete a los estudiantes en alguna actividad intelectual superior; los anima a comparar, aplicar, evaluar, analizar y sintetizar, pero nunca solo a escuchar y recordar.

2. Enseñar es atraer la atención y mantenerla. Para ello hay que despertar la curiosidad de los estudiantes. Esto exigen un compromiso, el compromiso de ofrecer unas clases a las que vale la pena acudir.

3. Un enigma activa el razonamiento de los estudiantes. Hay que construir una serie de preguntas "complementarias" que servirán para avanzar en el conocimiento, ver conexiones, encontrar contradicciones.

4. Una herramienta útil para conocer los modelos mentales de los estudiantes son los cuestionarios. Estos no deben ser muy largos y sirven para conocer el punto de vista del estudiantado sobre un fenómeno, concepto, procedimiento... su manera de pensar sobre él. Lo ideal es que las preguntas sean abiertas y estimulantes.

5. Por último, el Diario del profesor es una herramienta muy útil para reflexionar sobre nuestra práctica docente, detectar dificultades que nos encontramos y adoptar decisiones fundamentadas y posibles.

Ciclos de Mejora en el Aula (2020). Experiencias de Innovación Docente de la US Esta obra se distribuye con la licencia Creative Commons 
Palabras Clave: Bioquímica, Metabolismo, Biomedicina, Docencia Universitaria, Experimentación Docente Universitaria.

Keywords: Biochemistry, Metabolism, Biomedicine, University Teaching, University Teaching Experimentation.

\section{Referencias bibliográficas}

Bain, K. (2007). Lo que hacen los mejores profesores universitarios. Valencia: Publicacions de la Universitat de València.

De Alba, N. y Porlán, R. (2017). La metodología de enseñanza. En R. Porlán (Coord.). Enseñanza universitaria. Cómo mejorarla (pp. 37-53). Madrid: Morata.

Finkel, D. (2008). Dar clase con la boca cerrada. Valencia: Publicacions de la Universitat de València.

Rivero, A. y Porlán, R. (2017) La evaluación en la enseñanza universitaria. En R. Porlán (Coord.), Enseñanza universitaria. Cómo mejorarla (pp.73-91). Madrid: Ediciones Morata.

Ciclos de Mejora en el Aula (2020). Experiencias de Innovación Docente de la US Esta obra se distribuye con la licencia Creative Commons 